\section{Life on the Rebound: Resilience Science, Extreme Events, and Coastal Resilience}

\section{Deborah M. Brosnan ${ }^{1 *}$}

${ }^{1}$ One Health Institute University of California Davis, USA

There is a fundamental tension between resilient ecosystems, shaped by extreme events, and humans need for sustainable resources in a changing world. This tension is best understood through ecological science, but best resolved through multi-disciplinary efforts.

Coastal resilience serves as a valuable unifying concept, but is in danger of losing core features from its roots in ecological science [1]. Resilient ecosystems are defined by three primary characteristics. They suffer periodic extreme events (e.g. hurricanes) which measurably affect natural resources from species composition and abundances, to the range of trophic and non-trophic interactions; in the aftermath they undergo a recovery process; and return to an ecosystem which closely resembles the original one. The frequency, intensity, and spatial scales of extreme events are integral to resilience. Among scientists, the concept has generated important ecologicalsocietal debates. In the aftermath of the SE Asia tsunami controversy erupted over whether to restore disaster- damaged coral reefs, with some advocating for restoration and others arguing that reefs, having evolved with tsunamis, would rebound if left alone.

The disturbance-resilience interaction has been a driver in human settlement and behaviors. Communities have historically congregated on deltas where the cycles of flooding and storms so destructive to human life are also responsible for high productivity and ample natural resources that sustain life. A key challenge is how we will manage these opposing forces into the future.

Decision-makers, unfamiliar with ecological resilience, and seeking to build stability for humans can easily equate resilience with resistance. This can lead to unattainable expectations from nature, and to habitat restoration projects based on a desire to build resistant habitats, rather than resilient ones. Factoring-in ecosystem resilience requires an approach that is anathema to human's natural desire for greater stability.

Scientists can and do contribute to the global dialogue and solutions through basic scientific research, multi-disciplinary research, and by integrating results into decision-making. All three activities are needed. As coastal resilience becomes more multidisciplinary there is an accompanying need to strengthen the scientific underpinnings of ecological resilience by encouraging and supporting basic research in disturbances and extreme events. Are there key patterns and drivers that are common across different coastal and marine ecosystems? How do human activities interact with the drivers of resilience? Humans can alter the frequency, intensity, biological impact and nature of disturbances. Are there general patterns that can point to more global understanding of resilience and vulnerability, and which in turn can create solutions that are transferable and widely applicable.

One key area where ecological resilience and disturbance science is rarely incorporated but urgently needed is in Disaster Risk Reduction [2]. Disaster response professionals have developed tools to evaluate and respond to human and infrastructural needs in natural disasters from tsunamis to volcanoes. However, even though the effects of these events are frequently mediated by natural resources, and human suffering is exacerbated by damaged or scarce natural resources, the planning, response, recovery and mitigation framework that has proven so effective in addressing direct human needs does not incorporate ecosystems or ecosystem resilience.

Coastal resilience is a valuable concept that allows for interdisciplinary collaboration and for communication with decisionmakers. The drawback is that without scientific anchoring, it is at risk of becoming too vague or misunderstood. This concern is not new $[3,4]$. As coastal and marine ecosystems face a suite of natural and human-induced stressors, multi-disciplinary efforts are essential. Building a strong pillar of ecological science to support these efforts remains key. Human's ability to manage novel and combined stressors rests foremost on understanding the natural dynamics of resilient ecosystems, how humans interact with the drivers of resilience, and clear communication on resilience as a scientific principle and as a solution.

\section{References}

1. Holling CS (1973) Resilience and stability of ecological systems. Annu Rev Ecol Syst 4: 1-23

2. Meadows D (2008) Lessons for minimizing impacts to coral reef and other ecosystems from the 2004 tsunami. AM FISH S S 325-342.

3. Klein RJT, Nichols RJ, Thomalla F (2003) Resilience to natural hazards: How useful is this concept? Global Environmental Change Part B: Environmental Hazards 5: 35-45.

4. Strunz S (2012) Is conceptual vagueness an asset? Arguments from philosophy of science applied to the concept of resilience. Ecological Economics 76: 112-118. 